\title{
Susceptibility Weighted Imaging
}

National Cancer Institute

\section{Source}

National Cancer Institute. Susceptibility Weighted Imaging. NCI Thesaurus. Code

C121377.

An imaging technique that uses phase differences between tissues due to differences in magnetic susceptibilities to produce an enhanced contrast magnitude image. These phase differences can provide excellent contrast between gray matter and white matter, iron-laden tissues, venous blood vessels, and other tissues with susceptibilities that are different from the background tissue. The technique is commonly used for imaging in cases of traumatic brain injuries and for high resolution brain venographies. 